\title{
Ultrasonic Piezoelectric Motor with Intrinsic Torque Measurement - First Results
}

\author{
Pott, Peter; Matich, Sebastian; Schlaak, Helmut \\ Technische Universität Darmstadt, Institut für Elektromechanische Konstruktionen \\ Merckstraße 25, 64283 Darmstadt, Germany
}

\section{Problem}

Piezoelectric actuators are used, when small-scale applications with high torque demands at low speeds are of interest. Here, the inverse piezoelectric effect is used. It describes the ability of certain materials to change their geometry in an electric field [1]. The displacement is about $0.1 \%$ of the original length of the material, so for macro-scale movements, these small displacements have to be augmented or added up. Three different approaches can be distinguished: Lever-augmented stacks, stepping actuators, and oscillating actuators.

The first group consists of stacked piezoelectric elements integrated into a lever structure that provides an enlargement of the stroke [2-3]. The second group provides discrete steps as a sequence of clamping and pushing [4-6], sticking/slipping movements [7-8], or geared structures [9-10]. Finally, the third and largest group primarily uses the magnification of the amplitude near or at the resonance frequency of the oscillating structure. By adding up the derived stroke rather fast movements up to $1 \mathrm{~m} / \mathrm{s}$ and high thrust forces up to $30 \mathrm{~N}$ can be achieved [5].

The most common type is the travelling wave actuator introduced by Sashida in 1982 [11]. This is widely used for example in camera lenses [12] and shutters [13]. In addition, a variety of other principles is available [1, 14-15], that also use the inverse piezoelectric effect but involve other wave forms and make use of two different vibration modes (e.g. the system by Elliptec [16], Fleischer [17], or Uchino [18]. One major field of application for piezoelectric actuators is (micro- or nano-) positioning. Here, two bodies have to be moved in relation to each other with very high precision and repeatability. When stacks or augmented stacks are used, the hysteresis of the piezoelectric material can be compensated by controlling the charge rather than the voltage on the electrodes. For stepping motors or ultrasonic motors an additional position feedback is needed. However, when a force-controlled movement is needed in a system driven by an ultrasonic motor, external force or torque sensors are necessary, that affect the systems resonance behaviour and produce additional effort and costs. Force- or torque-measurement is useful for example when dealing with elastic material, for the design of micro-scale material testing machines, or in general, when closed-loop force control is needed in micro-mechatronic systems. The direct piezoelectric effect describes the fact, that when mechanical stress is applied to piezoelectric material, a voltage can be measured at the electrodes. So this material can be used as a force sensor. When both effects - the direct as well as the inverse piezoelectric effect - are used, self-sensing actuators can be built. These are widely used for adaptronic systems and vibration control [19-21], where sub-resonant oscillations in a frequency range well below $20 \mathrm{kHz}$ are of main interest. For the case of sensing in resonant or ultrasonic actuators, model-based approaches are described [22-23]. In many technical applications the actual torque provided by an actuator is of interest. Torque sensing, however, tends to be costly and complex as data and energy transmission to the rotating part is necessary in many cases [24]. When it comes to small-sized high-torque but low-velocity actuation systems ultrasonic piezoelectric actuators can be used. Here, high power density and noise-less operation can be provided.

In this paper we present an ultrasonic piezoelectric motor with intrinsic force sensing capabilities that provides high power density, high torque and a signal linearly correlated to the torque output, without the need of an additional sensor element.

\section{Technical Realisation}

The actuator (fig. 1) consists of a stainless steel bar $\left(50 \times 5 \times 5 \mathrm{~mm}^{3}\right)$ that is forced to resonant longitudinal vibration by a pre-stressed piezo element ( $\mathrm{Pz} 26,5 \times 2 \times 2 \mathrm{~mm}^{3}$; Noliac A/S, Kvistgaard, Denmark,) at $22.3 \mathrm{kHz}$. The stator bar is attached to leaf springs, to damp bending modes. The $\mathrm{Al}_{2} \mathrm{O}_{3}{ }^{-}$ rotor is mounted in a way that the bar can be tangentially pressed to it by a second piezo stack (PSt 150/5x5 / 7; Piezomechanik GmbH, Munich, Germany). By applying a two-phase signal to the piezo elements the friction contact is forced into the well-known elliptic movement and drives the rotor [25]. The piezo elements were driven by a LE 150/100EBW amplifier by Piezomechanik GmbH, Munich, Germany 
(driving piezo) and a AVB 250/30P amplifier by D*ASS GmbH, Saarbrucken, Germany (pressing piezo), respectively. For experimental reasons, a variable pre-stress can be applied by adding different weights radially to the rotor axis. To do so, the rotor axis is mounted in a seesaw-like mechanical set-up.

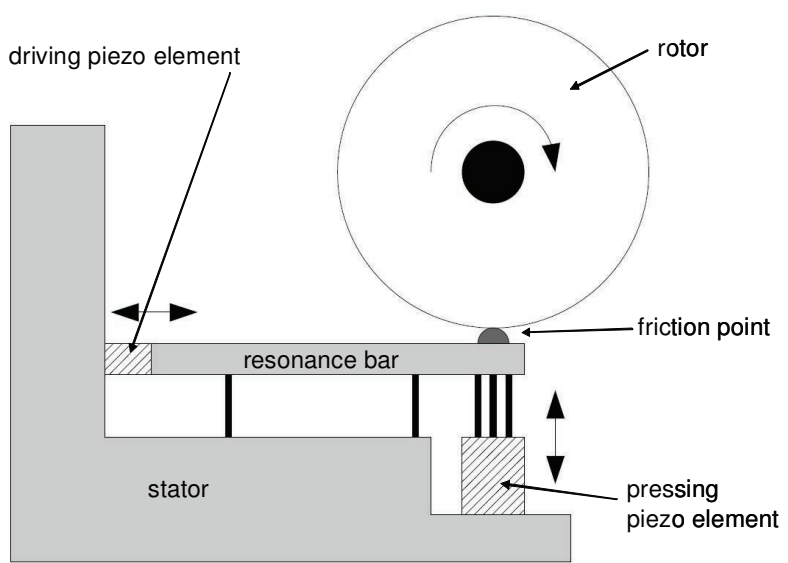

Fig. 1: Mechanical set-up of the resonant piezoelectric actuator with intrinsic torque measurement. Two piezoelectric elements driven by a phase-shifted voltage lead to an elliptic movement of the friction point (hardened steel) that forces the rotor to rotate.

The external torque is applied by lifting weights $m_{\text {lifted }}$ from a special set ranging from $1 \mathrm{~g}$ to $200 \mathrm{~g}$ that can be arbitrarily combined and added. The weights are lifted by means of a hoist with a $r_{\text {Hoist }}=15 \mathrm{~mm}$. The whole mechanical set-up is mounted to a vibration-damping table in a way that six full revolutions can be achieved and transient effects can decay.

The angle of rotation is measured by a HEDS 5500 encoder by Agilent Technologies (Santa Clara, CA, USA).

The lifted weight leads to a torque of

$$
\tau=m_{\text {lifted }} \cdot g \cdot r_{\text {Hoist }}
$$

acting on the rotor $\left(r_{\text {Rotor }}=15 \mathrm{~mm}\right)$ axle. Via the friction contact, this torque is held up by a static tangential force in the stator bar

$$
F_{\text {static,tngential }}=\frac{\tau}{r_{\text {Rotor }}} \text {. }
$$

This force leads to a torque-dependant bias in the signal provided by the driving piezo. Here, the piezoelectric effect causes a charge transfer - and thus a voltage $\left(U_{C M}\right)$ - that is proportional to the force applied. $U_{C M}$ can be measured by a circuit shown in the inset of fig. 2 .

To assess this voltage a measurment was realized. A standard PC hosted a RIO-PCI-7830R card by National Instruments (Austin, TX, USA) that provided four AD input channels $(200 \mathrm{kS} / \mathrm{s})$ and four DA output channels (1 MS/s). A Virtex-II-FPGA by Xillinx (San Jose, CA, USA) was also provided for realtime computations. For signal conditioing before the digitalization, the signal was amplified and low-pass filtered by an espacially developed analogue circuit. In the digital domain two filter principles were assessed: IIR Butterworth 4th ord filtering with $1 \mathrm{~Hz}$ cut-off frequency and FIR-filtering (type 1 with $\mathrm{n}=2000$ and a cut-off frequency of $0.25 \mathrm{~Hz}$ ) were applied.

To derive a driving signal, direct signal synthesis was used on the measurement card. The complete measurement set-up was controlled by a LabView program designed for the purpose. Here, a frequencysweep to detect the actual resonance frequency of the stator bar was performed prior to the actual experiments.

The actual experiment was the assessment of the characteristic curve rpm over torque and torque signal over torque. To achieve this, the ultrasonic actuator ran at idle speed for 4 minutes to allow temperaturedepending transient effects to settle. After that the experimentator only had to apply the different weights (50 g. $100 \mathrm{~g}, 150 \mathrm{~g}, 200 \mathrm{~g})$ to the thread coiled by the hoist. Six revolutions were assessed for each weight step. 


\section{Results}

Fig. 2 shows a step curve of the actual voltage of the measurement circuit after IIR filtering (grey line). The frequency of the signal correlates with the actual rotations. So it can be also seen, that for each load step six rotations were measured. The black line shows the signal after FIR filtering, that was applied for signal analysis.

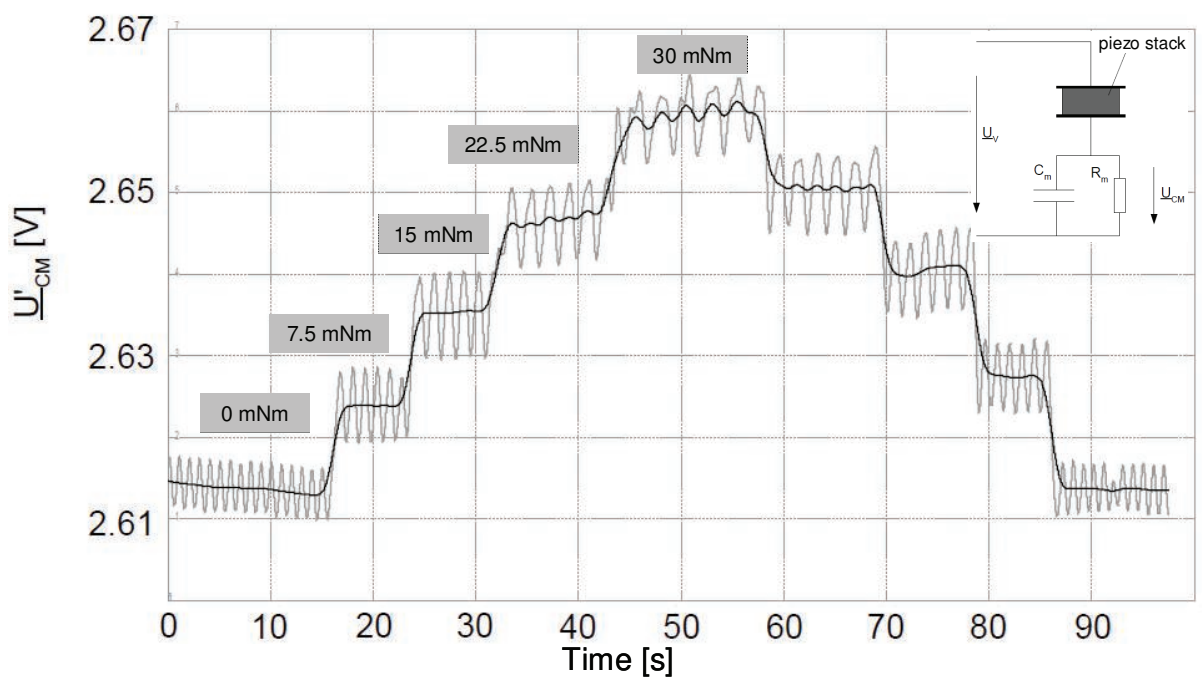

Fig. 2: Measured and filtered output of the measurement circuit (inset). The correlation of the signal with the output torque can be clearly seen. Also, the velocity of the actuator is observable by analysing the frequency of the signal.

Fig. 3 finally shows the actuator's characteristic curve and the correlation of the torque signal with the actual torque. The black line shows the torque-related signal $U_{C M}$ while the dotted line shows the dependency of rotating speed and torque. The torque-related signal provides a sensitivity of about $1.5 \mathrm{mV} / \mathrm{mNm}$. However, it is obvious, that the torque-related signal is affected by a hysteresis of about $10 \%$ and also the rotating speed showed some non-linear behaviour.

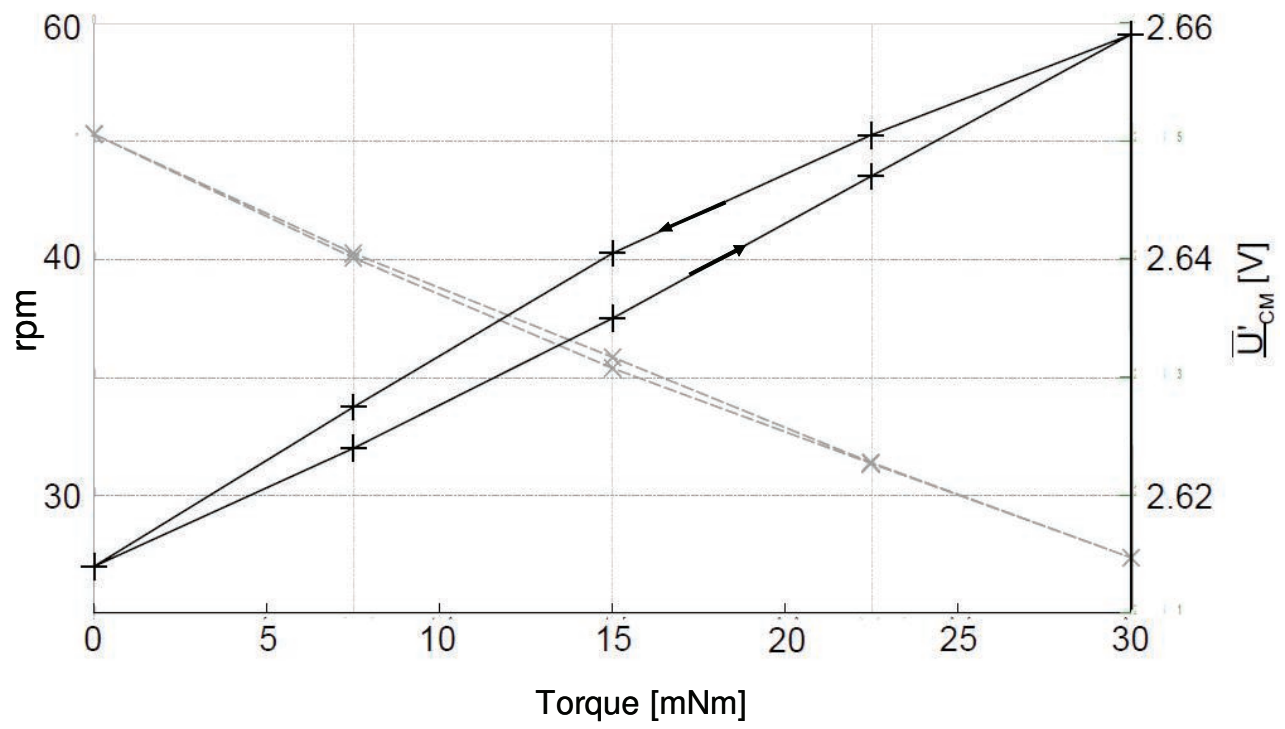

Fig. 3: Characteristic curve of the motor (grey line) and output-signal of the measurement circuit (black line). A hysteresis of about $10 \%$ is obvious.

\section{Discussion}

We were able to show, that the derivation of a torque-related signal in a piezoelectric ultrasonic actuator driven at resonance is possible with a good sensitivity of $1.5 \mathrm{mV} / \mathrm{mNm}$. Our first experiments with a laboratory prototype however showed a number of drawbacks that are related to the experimental character of the actuator. Especially the excessive wear of the hardened steel/ $/ \mathrm{Al}_{2} \mathrm{O}_{3}$ ceramics friction contact limits the number of consecutive tests and the lifetime of the actuator to about 30 minutes. Also, the heat generation in the piezoelectric elements leads to numerous non-linear effects. Some of these 
thermal effects can be compensated by carefully choosing the electronic devices in the measurement circuit as described e.g. in [19] for sub-resonant self-sensing piezo actuators. However, during the experiments we experienced some $10 \%$ hysteresis in the signal, so the final goal- a closed-circuit forcecontrol of an actuation system - is not yet achievable.

Future work will comprise the optimization of the electromechanical set-up (signal quality, sensitivity, and mechanical wear) and the build-up of an embedded system for signal analysis and force/velocity-control of the actuator.

\section{Acknowledgement}

The work is funded by the LOEWE-AdRIA project and the state government of Hesse, Germany

\section{References}

[1] K. Uchino.Piezoelectric Actuators and Ultrasonic Motors. (1996), Springer, Heidelberg

[2] C. Belly, F. Claeyssen, R. Le Letty, T. Porchez, Benefits from Amplification of Piezo Actuation in Inertial Stepping Motors and Application for High-performance Linear Micro Motors. Proc. of the ACTUATOR, Bremen, Germany (2010) 198-201

[3] Cedrat Group, Homepage, http://www.cedrat.com/en/mechatronic-products/actuators/apa.html, Zugriff am 16.06.2009.

[4] Piezomotor SA, Homepage, www.piezomotor.com, Zugriff am 16.06.2009.

[5] Physikinstrumente, Homepage, www.physikinstrumente.com, Zugriff am 16.06.2009.

[6] T. Galante, J. Frank, J. Bernard, W. Chen, G. A. Lesieutre, G. H. Koopmann, Design, Modeling, and Performance of a High Force Piezoelectric Inchworm Motor. Journal of Intelligent Material Systems and Structures, 10, 12 (1999), 962-972

[7] Konica Minolta, Piezoelectric supersonic linear actuator, http://www.konicaminolta.com/about/research/core technology/picture/antiblur.html, Zugriff am 22.09.2009.

[8] T. Morita, R. Yoshida, Y. Okamoto, M. K. Kurosawa, T. Higuchi, A Smooth Impact Rotation Motor Using a Multi-Layered Torsional Piezoelectric Actuator. IEEE Transactions on Ultrasonics, Ferroelectrics, and Frequency Control, 46, 6 (1999), 1439-1445

[9] U. Jungnickel, D. Eicher, H. F. Schlaak, Miniaturised micro-positioning system for large displacements and large forces based on an inchworm platform. Proc. of the ACTUATOR, Bremen, Germany (2002) 684-687

[10] A. Kappel, B. Gottlieb, T. Schwebel, C. Wallenhauer, PAD - A Piezoelectric Actuator Drive. Proc. of the ACTUATOR, Bremen, Germany (2006) 457-460

[11] T. Sashida, Motor device Utilizing Ultrasonic Oscillation, Patent-Nr.: JP 58-148682, 1982.

[12] T. Sashida, Mech. Automation Japan, 12, 15 (1983), 31

[13] Minolta Camera, Product Catalog "Mac Dual I, II". 1989.

[14] K. Uchino, Piezoelectric Actuators 2006. J Electroceram, 20, (2006), 301-311

[15] H. Janocha.Unkonventionelle Aktoren: Eine Einführung (2010), Oldenbourg (Munich),

[16] Elliptec Resonant Actuator AG, Homepage, http://www.elliptec.com, Zugriff am 22.06.2009.

[17] M. Fleischer, D. Stein, H. Meixner, New Type of Piezoelectric Ultrasonic Motor. IEEE Transactions on Ultrasonics, Ferroelectrics, and Frequency Control, 36, 6 (1989), 614-619

[18] K. Uchino, K. Kato, M. Tohda, Ultrasonic linear motors using a multilayered piezoelectric actuator. Ferroelectrics, 87, 1 (1988), 331-334

[19] L. Jones, E. Garcia, H. Waitest, Self-sensing control as applied to a PZT stack actuator used as a micropositioner. Smart Mater. Struct., 3, 2 (1994), 147-156

[20] B. Ko, B. H. Tongue, Acoustic Control using a Self-Sensing Actuator. Journal of Sound and Vibration, 187, 1 (1995), 145-165

[21] J. J. Dosch, D. J. Inman, E. Garcia, A Self-Sensing Piezoelectric Actuator for Collocated Control. Journal of Intelligent Material Systems and Structures, 3, (1992), 166-185

[22] F. Giraud, B. Semail, A Torque Estimator for a Traveling Wave Ultrasonic Motor-Application to an Active Claw. IEEE Transactions on Ultrasonics, Ferroelectrics, and Frequency Control, 53, 8 (2006), 1468-1477

[23] J. Maas, T. Sculte, N. Fröhleke, Model-Based Control for ultrasonic motors. IEEE Transactions on Ultrasonics, Ferroelectrics, and Frequency Control, 5, 2 (2000), 165-180

[24] W. J. Fleming, Automotive Torque Measurement: A Summary of Seven Different Methods. IEEE Transactions on Vehicular Technology, 31, 3 (1982), 117-124

[25] S. Devos, W. Van de Vijver, D. Mesonero-Romanos Vivanco, D. Reynaerts, H. Van Brussel, Piezoelectric Motors with a Stepping and a Resonant Operation Mode. Proc. of the ACTUATOR, Bremen, Germany (2004) 